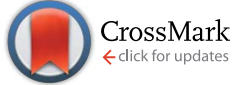

Cite this: RSC Adv., 2015, 5, 82564

\title{
Thin water films and magnesium hydroxide fiber growth $\dagger$
}

\begin{abstract}
Amir Gheisi, ${ }^{a}$ Andreas Sternig, ${ }^{a}$ Günther J. Redhammer ${ }^{b}$ and Oliver Diwald ${ }^{* b}$
Thin films of water covering highly dispersed metal oxides can give rise to the spontaneous and spatially controllable growth of hydroxide fibers in ambient air. Knowledge about the underlying formation mechanisms is key to the rational development of metal oxide nanomaterials and associated microstructures. We used $\mathrm{SiCl}_{4}$ as a water free chlorine ion source for the surface functionalization of $\mathrm{MgO}$ nanocubes and explored their subsequent transformation into magnesium oxychloride $\mathrm{Mg}_{3}(\mathrm{OH})_{5} \mathrm{Cl} \cdot 4 \mathrm{H}_{2} \mathrm{O}$ fibers upon contact with water vapor. Specifically we show how the temperature of the functionalization process and material dispersion control the reaction pathway that can lead to very different products like $\mathrm{Mg}(\mathrm{OH})_{2}, \mathrm{MgCl}_{2} \cdot 6 \mathrm{H}_{2} \mathrm{O}$, or $\mathrm{Mg}_{3}(\mathrm{OH})_{5} \mathrm{Cl} \cdot 4 \mathrm{H}_{2} \mathrm{O}$ and delineate a reaction mechanism. Lessons to be learned from this unique route to form hydroxide fibers under ambient conditions can be applied to a variety of microstructural evolution processes that involve metastable solids and superficial water acting both as a reactant and as a reaction medium for the hydration of metal oxide particles.
\end{abstract}

Received 6th September 2015

Accepted 21st September 2015

DOI: $10.1039 / c 5 r a 18202 f$

www.rsc.org/advances

\section{Introduction}

The implementation of metal oxides and hydroxides into functional and structural materials requires knowledge about their growth and stability in changing chemical environments. These will never be understood without the examination of specific aspects of that complexity. In this regard, tailored particle systems with interface properties which are accessible to experimental methods are indispensable model systems. ${ }^{1,2}$

Most growth processes yielding oxide nanoparticles occur under non-equilibrium conditions. ${ }^{3,4}$ Resulting solids are metastable, highly dispersed and, as such, subject to their interface and the chemical composition of the surrounding gas or liquid phase. Under ambient conditions and in humid environments thin-film water is ubiquitous ${ }^{2,5,6}$ and nanomaterials become instantaneously covered by water films with thicknesses ranging between one molecular layer to few nanometers. ${ }^{7}$ While the water-mineral oxide chemistry has received much attention in earth and environmental science ${ }^{6,8}$ as well as in surface and materials science ${ }^{7,9-11}$ significantly less attention has been paid to interfacial water in the field of nanomaterials

${ }^{a}$ Institute of Particle Technology, Universität Erlangen-Nürnberg, Germany ${ }^{b}$ Department of Materials Science and Physics, Paris-Lodron University Salzburg, Austria. E-mail: oliver.diwald@sbg.ac.at

$\dagger$ Electronic supplementary information (ESI) available: Supporting powder X-ray diffraction data with the results of Rietveld refinements and additional electron microscopy images together with a table of estimated surface coverages of $\mathrm{SiCl}_{4}$ and $\mathrm{O}_{2}$ on the different types of nanocrystalline $\mathrm{MgO}$ samples. See DOI: $10.1039 / \mathrm{c} 5 \mathrm{ra} 18202 \mathrm{f}$ chemistry. This is surprising if one considers that thin water films can serve as a reaction medium for the growth and assembly of oxide nanostructures. ${ }^{12,13}$

We recently discovered the spontaneous formation of magnesium oxychloride $\left(\mathrm{Mg}_{3}(\mathrm{OH})_{5} \mathrm{Cl} \cdot 4 \mathrm{H}_{2} \mathrm{O}\right)$ fibers which grow out of MgO-based nanoparticle agglomerates. ${ }^{14}$ This process occurs under ambient conditions and starts from $\mathrm{MgO}$ nanocubes the surfaces of which were previously contacted with chlorine. Prior to fiber growth both the synthesis and the functionalization procedure of the metal oxide nanoparticles exclusively involved the solid-gas interface and were performed under dry conditions. The critical impact of materials and process parameters during functionalization, such as the required degree of dispersion or the temperature of adsorption and surface functionalization has remained unknown to date. In this study, we identified their decisive impact on the entire transformation process to gain insights into the underlying growth mechanism and, ultimately, to control microstructure development. Moreover, the challenge of achieving compositional homogeneity over the entire nanoparticle ensemble is at the focus of this study. The detailed exploration of the here presented solid-liquid-gas interface reaction involves surface functionalized non-equilibrium solids and thin liquid water films serving as reaction medium and solvent. The study provides important insights for the synthesis and functionalization of oxide nanomaterials. Moreover, it also connects to key steps in the interface chemistry of mineral binders and ceramics which is determining for their later performance. 


\section{Experimental section}

\subsection{Chemical vapor synthesis of $\mathrm{MgO}$}

$\mathrm{MgO}$ nanoparticles were produced via a chemical vapor synthesis (CVS) technique which is based on controlled combustion of metal vapor within a flow reactor system. ${ }^{\mathbf{1 5 , 1 6}}$ For further materials processing the samples are transferred through ambient air into a quartz glass tube. In order to generate cubically shaped nanocrystals and to guarantee bare particle surfaces, thermal sample activation via vacuum annealing is employed. A typical procedure utilized for dehydration, dehydroxylation ${ }^{\mathbf{1 7 , 1 8}}$ and removal of carbon-based surface contaminants is as follows: as-synthesized powders are heated to $T=1123 \mathrm{~K}$ in high vacuum with a rate of $5 \mathrm{~K} \mathrm{~min}^{-1}$ and then are brought into contact with $10 \mathrm{mbar}_{2}$ at this temperature for 10 minutes. Subsequently, the temperature is raised to $1173 \mathrm{~K}$ and, at pressures of $p<5 \times 10^{-6} \mathrm{mbar}$, kept at this temperature for $1 \mathrm{~h}$ before cooling to room temperature. ${ }^{19}$ As revealed by Transmission Electron Microscopy CVS MgO consists of highly dispersed monocrystalline nanocubes with a high portion of edge and corner features (Fig. S1a, ESI $\dagger$ ). ${ }^{20}$ In addition to CVS MgO, we also employed commercial nanocrystalline MgO (529699 Aldrich) for reference experiments (Fig. S1 and S2 $\dagger$ ). This material can be characterized as an assembly of less regular shaped particles (Fig. S1b, ESI $\dagger$ ). Whereas the Scanning Electron Microscopy (SEM) images of CVS MgO nanoparticles reveal a fine-grained material with an open and porous secondary structure (Fig. S1c, ESI $\dagger$ ), micrographs related to commercial $\mathrm{MgO}$ particles reveal their more coarse grained nature. Respective particles do not exhibit any characteristic and prevailing shape.

XRD confirms for both types of $\mathrm{MgO}$ powders the exclusive presence of crystalline particles with periclase structure (Fig. S2, ESI $\dagger$ ). The average crystallite sizes, as determined from peak broadening using the Scherrer equation, ${ }^{21,22}$ correspond to $6 \pm 1$ $\mathrm{nm}$ and $35 \pm 1 \mathrm{~nm}$ for CVS $\mathrm{MgO}$ and commercial $\mathrm{MgO}$, respectively. While the measured specific surface area of CVS $\mathrm{MgO}$, which corresponds to $S_{\mathrm{BET}}=300 \pm 20 \mathrm{~m}^{2} \mathrm{~g}^{-1}$, is perfectly consistent with the XRD derived specific surface area, the situation is different for commercially available $\mathrm{MgO}$ : in comparison to a XRD derived value of $S_{\mathrm{XRD}}=50 \mathrm{~m}^{2} \mathrm{~g}^{-1}$ we determined via $\mathrm{N}_{2}$ sorption analysis a value of $S_{\mathrm{BET}}=12 \pm 1 \mathrm{~m}^{2} \mathrm{~g}^{-1}$ which is lower by a factor of five. This discrepancy points to polycrystalline grains exhibiting a large fraction of intergranular solid interface area that is not susceptible to $\mathrm{N}_{2}$ adsorption. Both CVS and commercial $\mathrm{MgO}$ were processed equally prior to functionalization and growth studies described in this work.

\section{2. $\mathrm{MgO}$ surface functionalization via $\mathrm{SiCl}_{4} / \mathrm{O}_{2}$ exposure}

$\mathrm{MgO}$ is exposed to $\mathrm{SiCl}_{4}$ and $\mathrm{O}_{2}$ in a cyclic process. The schematic of the setup used for this process is shown in Fig. 1a. Prior to adsorption $\mathrm{SiCl}_{4}$ is cleaned employing the freeze-pump-thaw method. The base pressure of the sample tube at room temperature is less than $p=10^{-5}$ mbar. In addition to adsorption experiments at room temperature we also

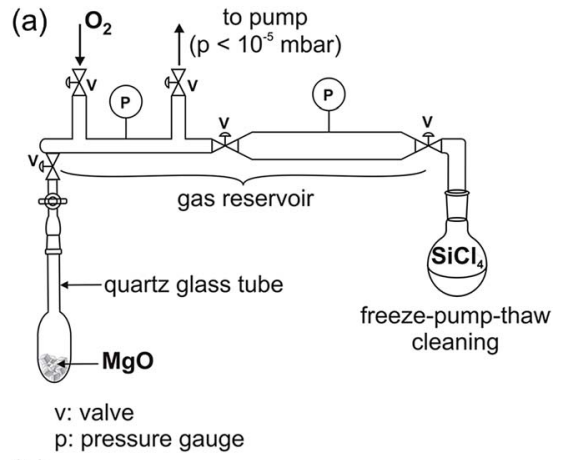

(b)

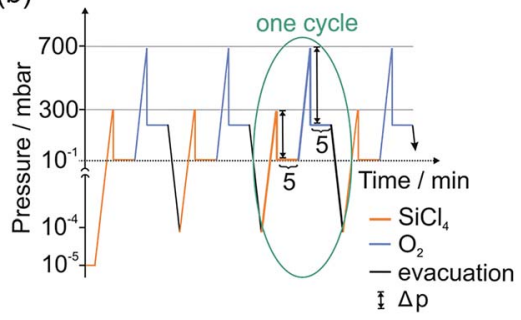

Fig. 1 (a) Schematic illustrating the setup used for the $\mathrm{MgO}$ exposure to $\mathrm{SiCl}_{4} / \mathrm{O}_{2}$ and (b) typical process cycle employed for this functionalization approach. The time-dependent application of $\mathrm{SiCl}_{4}$ and $\mathrm{O}_{2}$ partial pressures (b) was used for the functionalization cycles of $\mathrm{MgO}$ nanocube ensembles at $T=298 \mathrm{~K}$ or at $77 \mathrm{~K}$.

performed experiments at $77 \mathrm{~K}$. In these cases the lower part of the glass tube containing the MgO nanocube powder (adsorbent) was immersed into a liquid nitrogen bath in order to keep $77 \mathrm{~K}$ as a constant temperature of adsorption. Samples which were functionalized in this way will be designated in the following as low temperature adsorption (LTA) samples.

For a typical functionalization approach we exposed approximately $200 \mathrm{mg}$ of $\mathrm{MgO}$ nanocube powder within a first cycle for 5 minutes to $\mathrm{SiCl}_{4}$ vapor (with a vapor pressure of 300 mbar at room temperature) and after subsequent pumping subjected the powder to $\mathrm{O}_{2}$ atmosphere ( $p=700 \mathrm{mbar}$ ) for another period of 5 minutes. Gaseous reactants that did not adsorb on the particle surface are removed upon subsequent pumping to a base pressure of $p<10^{-4}$ mbar and new reactant molecules are supplied to the powder sample in the course of each subsequent cycle. The results presented in this study were obtained on $\mathrm{MgO}$ nanocube powders which were subjected to 6cycles of room (RTA) or low temperature (LTA) adsorption. The $6^{\text {th }}$ cycle is completed by sample evacuation to the base pressure of $p<10^{-4} \mathrm{mbar}$ at $298 \mathrm{~K}$.

\section{Results and discussion}

\subsection{Surface functionalization of $\mathrm{MgO}$ particles}

We used silicon tetrachloride $\left(\mathrm{SiCl}_{4}\right)$ as a carbon free chlorine source in order to achieve a compositionally homogeneous coverage with chlorine on the surfaces of the $\mathrm{MgO}$ particle ensemble. ${ }^{23}$ The surface coverages of CVS and commercial $\mathrm{MgO}$ that correspond to the applied $\mathrm{SiCl}_{4}$ and $\mathrm{O}_{2}$ pressures vary by a factor of 27 (Table S1, ESI $\dagger$ ). The respective gas phase 
functionalization was performed by different sequences of $\mathrm{SiCl}_{4} / \mathrm{O}_{2}$ admission either at $T=77 \mathrm{~K}$ (LTA Fig. $2 \mathrm{~b}$ ) or at $T=$ 298 K (RTA, Fig. 2c). We identified a particular sequence of reaction steps (Fig. 1b) as particularly robust approach and found that - depending on the temperature of adsorption - the functionalization process can follow entirely different reaction paths (Fig. 2): at room temperature, a strongly exothermic and poorly controllable process gives rise to a sample heterogeneity which is visible to the eye (see Fig. 2c). Different powder regions with particle agglomerates of varying quality and optical appearance are formed. Parts of the originally fluffy $\mathrm{MgO}$ nanocube powder (sample shown in Fig. 2a) become transformed into compact white flakes (c2 in Fig. 2). Both TEM analysis (right panel in the middle of Fig. 2) and analysis of the powder X-ray diffraction patterns (panel in the middle of Fig. 2) reveal that only a fraction of the starting material has become converted into the substantially larger grains that correspond to the crystalline $\mathrm{MgCl}_{2} \cdot 6 \mathrm{H}_{2} \mathrm{O}$ phase (Bischofite, c2, lower left panel of Fig. 2). (This part must originate from a water free and so far not isolated intermediate phase which

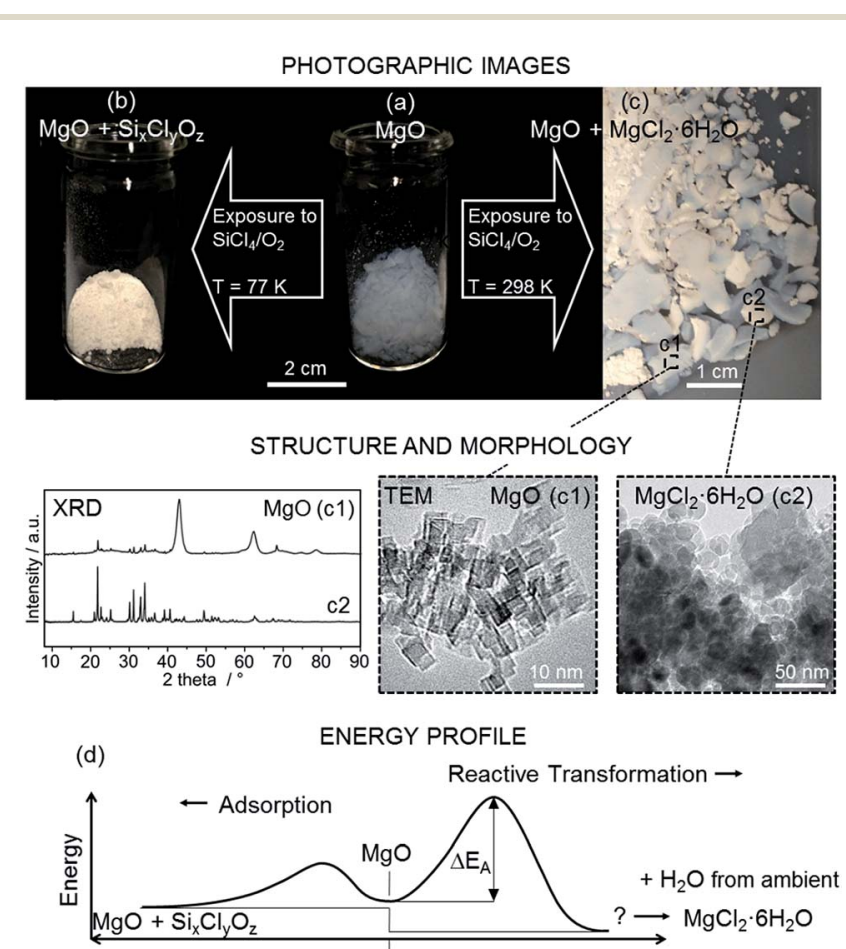

Fig. 2 Optical appearance and structural properties of chlorine functionalized $\mathrm{MgO}$ nanocube samples with different levels of compositional homogeneity. Exposure of an originally white and opalescent $\mathrm{MgO}$ nanocube powder (a) to $\mathrm{SiCl}_{4} / \mathrm{O}_{2}$ at $T=77 \mathrm{~K}$ produces a homogeneous white and compact powder (b). The inhomogeneous appearance of the sample in (c) arises from an identical sample treatment that was performed at $T=298 \mathrm{~K}$ and is due to the coexistence of two fractions: (c1) agglomerates of unreacted $\mathrm{MgO}$ nanocubes and (c2) parts of a more compact and deeply white powder with a XRD pattern which is specific to $\mathrm{MgCl}_{2} \cdot 6 \mathrm{H}_{2} \mathrm{O}$ grains (please find Rietveld analysis of the pattern in Fig. S3, ESI $\dagger$ ). The energy profile in the bottom panel underlines that adsorption at $77 \mathrm{~K}$ inhibits the strongly exothermic reactive transformation of $\mathrm{MgO}$ into $\mathrm{MgCl}_{2} \cdot 6 \mathrm{H}_{2} \mathrm{O}$ leads to $\mathrm{MgO}$ nanocubes with surface adsorbed $\mathrm{Si}_{x} \mathrm{Cl}_{y} \mathrm{O}_{z}$ (b). ${ }^{14}$ transforms within the time of the XRD measurement ( $<5$ hours) into $\mathrm{MgCl}_{2} \cdot 6 \mathrm{H}_{2} \mathrm{O}$.) The remaining fraction of the inhomogeneous powder has apparently not been affected by the chemical transformation process and consists of agglomerates of $\mathrm{MgO}$ nanocubes with periclase structure (c1 in Fig. 2).

For specimens isolated from the more compact regions of the powder sample (Fig. $3 \mathrm{c} 2$ ), phase analysis revealed magnesium dichlorhydrate $\mathrm{MgCl}_{2} \cdot 6 \mathrm{H}_{2} \mathrm{O}$ (Bischofite) as the predominant phase $(69 \%)$ together with forsterite $(28 \%)$ and periclase (3\%) as minor fractions (Fig. S3, ESI $\dagger$ ). The sample heterogeneity emerges directly after the $\mathrm{SiCl}_{4} / \mathrm{O}_{2}$ adsorption step, i.e. before the vacuum of the reaction cell (Fig. 1) was broken for sample transfer and further materials characterization. Apparently, water from the ambient, which is unavoidable in our current XRD set up, leads to the transformation of a so far unknown precursor phase into $\mathrm{MgCl}_{2} \cdot 6 \mathrm{H}_{2} \mathrm{O}$ (Fig. 2c2).

Earlier powder XRD and solid state ${ }^{29} \mathrm{Si}$ MAS NMR studies revealed that the LTA sample consists of $\mathrm{MgO}$ nanocubes that are covered with surface adsorbed chlorosiloxane species $\mathrm{Si}_{x^{-}}$ $\mathrm{Cl}_{y} \mathrm{O}_{z} \cdot{ }^{14}$ Despite their pronounced reactivity in the ambient, the chemical composition as well the nano-/microstructure of this metastable composite can be retained over weeks of storage in vacuum. As a major finding, the adsorption temperature represents the determining factor for the here described functionalization approach. In case of the room temperature adsorption (RTA) experiment the corresponding thermal energy in conjunction with the heat of reaction released allows the system to overcome the activation barrier $\Delta E_{\mathrm{A}}$ (Fig. 2d) towards formation of $\mathrm{MgCl}_{2} \cdot 6 \mathrm{H}_{2} \mathrm{O}$ (see XRD pattern and TEM image in Fig. 2c2) and forsterite (Fig. S3, ESI $\dagger$ ).

\subsection{Spontaneous fiber growth}

Due to the poor sample homogeneity of the RTA MgO powder sample (Fig. 2c) we subsequently concentrated on the LTA

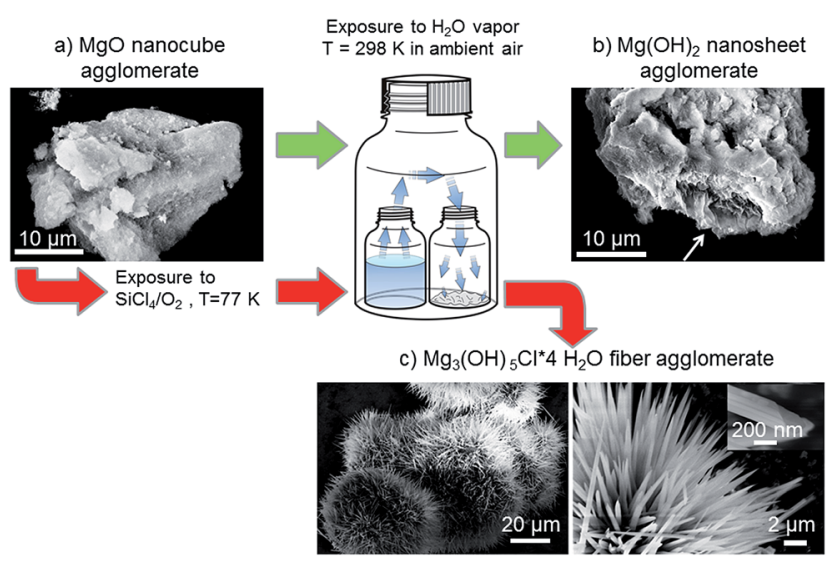

Fig. 3 Schematic illustrating the $\mathrm{H}_{2} \mathrm{O}$ vapor induced transformation of $\mathrm{MgO}$ nanocube powders into different microstructures, such as (a) $\mathrm{Mg}(\mathrm{OH})_{2}$ nanosheet agglomerates or (b) magnesium oxychloride fiber ensembles ( $c$, out of $\mathrm{Si}_{x} \mathrm{Cl}_{y} \mathrm{O}_{z}$ functionalized $\mathrm{MgO}$ ). The reaction system was kept at atmospheric pressure with $p\left(\mathrm{H}_{2} \mathrm{O}\right)=32 \mathrm{mbar}$ as equilibrium partial pressure of water at $298 \mathrm{~K}$. 
samples and characterized their response towards water vapor. The exceptional reactivity of $\mathrm{MgO}$ nanocubes, the surfaces of which have been functionalized with $\mathrm{Si}_{x} \mathrm{Cl}_{y} \mathrm{O}_{z}$ (Fig. 1c and 2b), ${ }^{14}$ gives rise to a novel and unexpected transformation process. Using separate reaction systems for the different experiments, we exposed functionalized and non-functionalized $\mathrm{MgO}$ nanocube powders to water saturated air at atmospheric pressure and for typical periods of 3 days or 14 days. A sketch of the experiments performed together with the characteristic microstructures obtained in the course of this room temperature reaction is provided in Fig. 3 .

The $\mathrm{MgO}$ particles are arranged in particle agglomerates (Fig. 3a). Scanning Electron Microscopy (SEM) measurements do not reveal any characteristic morphological features in the $\mu \mathrm{m}$ size range on these agglomerates. Room temperature exposure of bare $\mathrm{MgO}$ particle surfaces to water vapor produces thin lamellar features (e.g. see arrow in the upper right panel of Fig. 3) which are characteristic for $\mathrm{Mg}(\mathrm{OH})_{2}$ nanosheets, the crystallographic phase analysis of which is presented below. (A detailed microstructural and morphological description of these hydroxides can be found in ref. 19.) A completely new microstructural situation, however, originates from water contact of chlorosiloxane covered $\mathrm{MgO}$ nanocube agglomerates for exposure times $t \geq 3$ days $^{24}$ (lower panel in Fig. 3): assemblies of needle-like shaped crystals, which have grown radially out of the individual agglomerates are observed. We also checked for related microstructural transformation behavior on commercially available nanocrystalline low surface area $\mathrm{MgO}$ material $\left(S_{\mathrm{BET}}=12 \mathrm{~m}^{2} \mathrm{~g}^{-1}\right)$ which, prior to water vapor exposure, had been subjected to functionalization cycles identical to those applied to CVS MgO nanocubes (Table S1 in ESI $\dagger$ ). In this case XRD patterns (Fig. S4a, ESI $\dagger$ ) and SEM images (Fig. S4b, ESI $\dagger$ ) point to the exclusive formation of $\mathrm{Mg}(\mathrm{OH})_{2}$ and the absence of fiber growth or other additional crystallographic phases.

The different microstructures observed for pure and surface functionalized $\mathrm{MgO}$ nanocube ensembles after water vapor exposure (Fig. 3) are in line with crystallographic structure changes (Fig. 4).

Water vapor converts $\mathrm{MgO}$ nanocubes into $\mathrm{Mg}(\mathrm{OH})_{2}$ (Fig. 4a). However, the interaction between chlorosiloxane covered $\mathrm{MgO}$ nanocubes and $\mathrm{H}_{2} \mathrm{O}$ vapor gives rise to the $\mathrm{F} 5$ phase $\mathrm{Mg}_{3}(\mathrm{OH})_{5} \mathrm{Cl} \cdot 4 \mathrm{H}_{2} \mathrm{O}$ as concluded from the XRD powder pattern (Fig. 4c and S5 in ESI $\dagger$ ). ${ }^{\mathbf{1 4 2 6}}$ A graphical representation of this structure is provided in Fig. 4d. Importantly, in the course of all the experiments performed we have never obtained any evidence for magnesium oxychloride carbonatation which would result from $\mathrm{CO}_{2}$ uptake from the gas phase. ${ }^{27}$

For complementation of materials characterization we analyzed the LTA samples at the different stages of their transformation with TEM (Fig. 5). The granular contrast in Fig. 5a and $\mathrm{b}$ indicates a high degree of crystallinity of the particles after low temperature contact with $\mathrm{SiCl}_{4} / \mathrm{O}_{2}$ (Fig. 1b). Displaying sharp edges and corners their cubic habitus is essentially retained. Furthermore, consistent with the crystallite domain size the particle sizes, as determined by TEM analysis, remain in the range below $10 \mathrm{~nm} .{ }^{19}$ Irrespective from the retained primary particle properties, the agglomerates have become more compact (upper micrographs of Fig. 5a and b) in comparison to $\mathrm{MgO}$ nanocube powders before functionalization (Fig. S1a, ESI $\dagger$ ). ${ }^{19,28}$

From microscopy and XRD data in conjunction with the NMR evidence of siloxane formation on $\mathrm{MgO}$ surfaces ${ }^{\mathbf{1 4}}$ we infer that $\mathrm{SiCl}_{4} / \mathrm{O}_{2}$ adsorption (Fig. $1 \mathrm{~b}$ ) does not affect the primary particle properties but decreases their average distance and, by that, increases the powder density. This explains the changes in the scattering properties and, thus, the altered macroscopic optical appearance as revealed by the digital images in Fig. $2 \mathrm{~b}$. The larger $\mathrm{Mg}_{3}(\mathrm{OH})_{5} \mathrm{Cl} \cdot 4 \mathrm{H}_{2} \mathrm{O}$ fibers (Fig. 5) are arranged as bundles (inset in Fig. 5c) with diameters in the range between 100 and $300 \mathrm{~nm}$. After 14 days of $\mathrm{H}_{2} \mathrm{O}$ exposure we did not evidence residues of $\mathrm{MgO}$ nanocubes which previously had served as $\mathrm{Mg}^{2+}$ ion source for magnesium oxychloride fiber growth.
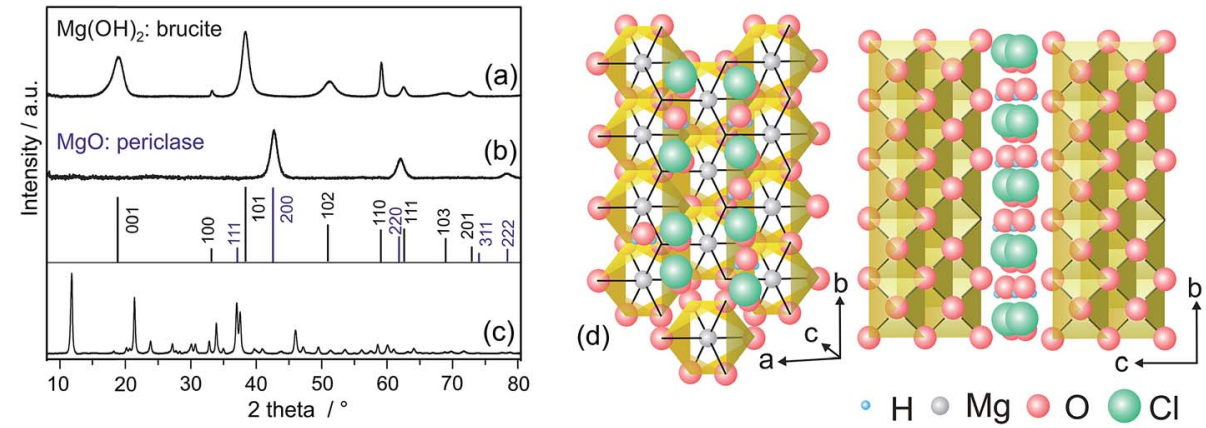

Fig. 4 Left panel: (a) powder XRD pattern of a MgO powder after storage in water-saturated air $\left(p\left(\mathrm{H}_{2} \mathrm{O}\right)=32 \mathrm{mbar}\right)$, (b) a chlorinated CVS MgO powder after storage in vacuum $\left(p<10^{-4} \mathrm{mbar}\right.$ ) and (c) a chlorinated CVS MgO powder after storage in water-saturated air $\left(p\left(\mathrm{H}_{2} \mathrm{O}\right)=32 \mathrm{mbar}\right)$. The measured XRD patterns are consistent with (a) the brucite phase $\mathrm{Mg}(\mathrm{OH})_{2}$ (black lines, JCPDS card \# 44-1482) and (b) periclase MgO (blue lines, JCPDS card \# 45-0946). Rietveld refinement of the pattern in (c) (Fig. S5 in ESI $†$ ) indicates a quantitative materials conversion into the F5 phase of magnesium oxychloride $\left(\mathrm{Mg}_{3}(\mathrm{OH})_{5} \cdot \mathrm{Cl} \cdot 4 \mathrm{H}_{2} \mathrm{O}\right)$. The right panel shows a graphical representation of the corresponding crystal structure as adopted from ref. 25. 

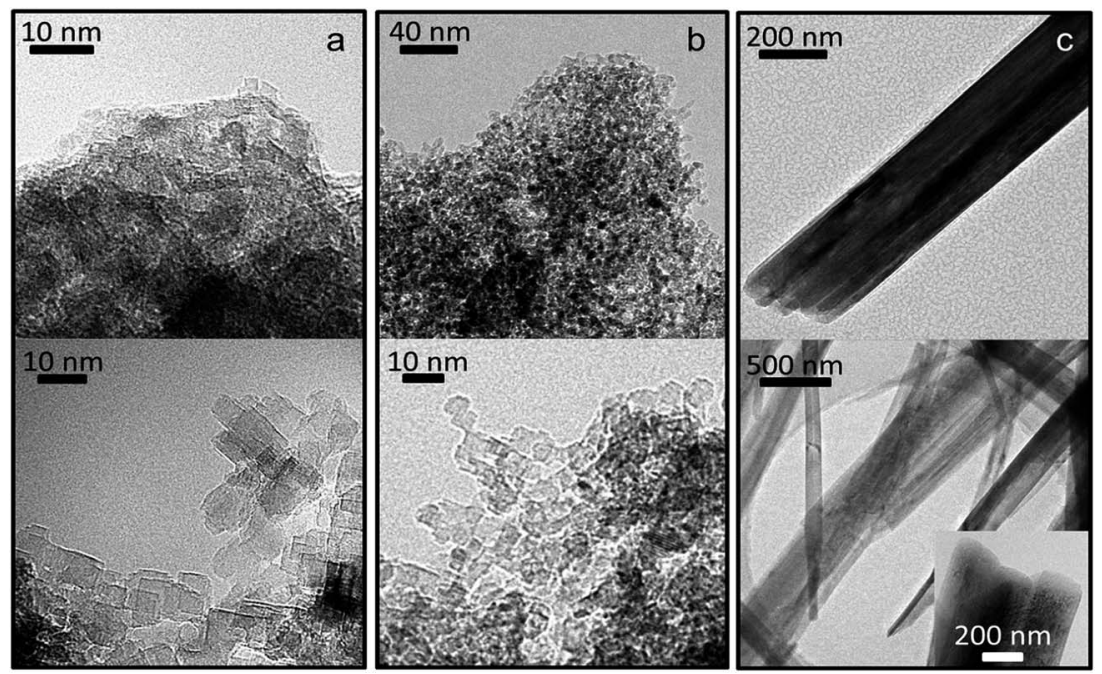

Fig. 5 Representative TEM images of (a) chlorinated MgO nanocubes; (b) chlorinated $\mathrm{MgO}$ nanocubes after storage in vacuum ( $p<10^{-4} \mathrm{mbar}$, 14 days) and (c) after 14 days of contact with water vapor $\left(p\left(\mathrm{H}_{2} \mathrm{O}\right)=32 \mathrm{mbar}\right)$.

\subsection{A gasphase transformation process with thin water films as liquid reaction medium}

We identified a novel transformation process that is based on the interaction between a water saturated gas phase and a highly dispersed metal oxide nanoparticle powder which was previously processed in water free environment. On oxide surfaces water adsorption occurs instantaneously when they get in contact with humidity. ${ }^{6}$ The reactivity of the chlorinated $\mathrm{MgO}$ nanocube ensemble is attributed to the presence of water multilayers as a confined liquid solvent medium for ions (Fig. 6). Inside these water films gradients related to the different ion concentrations enable the transport of $\mathrm{Mg}^{2+}, \mathrm{OH}^{-}$ and $\mathrm{Cl}^{-}$ions along the anisotropically growing structures. This sustains the growth process via diffusion and material precipitation at the top part of the growing fibers.

We used $\mathrm{SiCl}_{4}$ as a water free chlorine ion source ${ }^{23}$ and coadsorbed these molecules with $\mathrm{O}_{2}$ at low temperatures. Upon

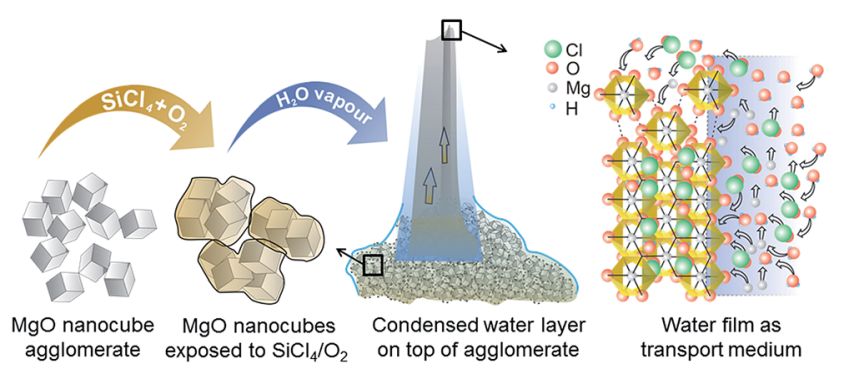

Fig. 6 Scheme illustrating the mechanism of fiber growth. Contact with water vapor leads to the instantaneous formation of condensed water films which sustain ion transport necessary to add new $\mathrm{Mg}_{3}(\mathrm{OH})_{5} \mathrm{Cl} \cdot 4 \mathrm{H}_{2} \mathrm{O}$ units to the top of the growing crystalline fibers. As concluded from the characteristic and well-characterized morphology of oxychloride fibers ${ }^{25}$ the [010] direction is proposed to be the growth direction with the (101) plane being subject to fast material addition and growth. subsequent sample warming to room temperature and under dynamic vacuum conditions, the $\mathrm{MgO}$ particle surfaces transform a fraction of activated $\mathrm{SiCl}_{4} / \mathrm{O}_{2}$ molecules into chlorosiloxane species that remain on the $\mathrm{MgO}$ nanocube surfaces. Comparison of the two types of nanocrystalline $\mathrm{MgO}$ samples, i.e. the CVS MgO nanocube powder (Fig. S1a and c, ESI $\dagger$ ) and commercially available MgO (Fig. S1b and d, ESI $\dagger$ ), shows clearly that the degree of dispersion is key to the transformation process (Fig. 3 and 6). On CVS $\mathrm{MgO}$, where reactive $\mathrm{SiCl}_{4} / \mathrm{O}_{2}$ adsorption (Fig. 1b) can occur, the estimated number of monolayer equivalents corresponds to approximately 1 and is by a factor of 28 lower as compared to commercially available nanocrystalline $\mathrm{MgO}$ (Table $\mathrm{S} 1, \mathrm{ESI} \dagger$ ). In the latter case the materials' dispersion is apparently too low to enable effective intermixture between $\mathrm{SiCl}_{4} / \mathrm{O}_{2}$ derived adsorbates, on the one hand, and activating $\mathrm{MgO}$ surface sites, on the other. As a consequence, $\mathrm{Si}_{x} \mathrm{Cl}_{y} \mathrm{O}_{z}$ production is negligible. The evacuation step at the end of each functionalization cycle (Fig. 1b) is performed during warm up and leads to the removal of all physisorbed and weakly bound $\mathrm{SiCl}_{4} / \mathrm{O}_{2}$ molecules. Since the concentration of surface activated chlorsiloxanes $\mathrm{Si}_{x} \mathrm{Cl}_{y} \mathrm{O}_{z}$ is insufficient to engage the reaction path towards magnesium oxychloride fiber formation, the exposure of this low surface area material towards $\mathrm{H}_{2} \mathrm{O}$ leads to the quantitative transformation of $\mathrm{MgO}$ into brucite (Fig. S4, ESI $\dagger$ ). An additional explanation relates to the complex reaction network which requires $\mathrm{Mg}-\mathrm{O}$ dissolution to enrich the liquid water film with $\mathrm{Mg}^{2+}$ ions. Size dependent dissolution effects ${ }^{19}$ as well as microstructural parameters which hamper mass transfer may eliminate the chance for magnesium oxychloride growth in case of the commercially available starting material. Silicon originating from $\mathrm{SiCl}_{4}$ is neither part of the crystalline product nor does it contribute to any other crystalline phase as concluded from the XRD measurements. We expect that after fiber growth amorphous $\mathrm{SiO}_{x}$ remains in the base region of the fiber assemblies. 


\section{Conclusions}

Agglomerated $\mathrm{MgO}$ nanoparticles can be functionalized with surface oxychlorides via the gasphase using $\mathrm{SiCl}_{4}$ as chlorine source. This produces high energy materials which, as a result of $\mathrm{H}_{2} \mathrm{O}$ vapor exposure, undergo spontaneous room temperature conversion into magnesium oxychloride fibers. Via the detailed exploration of the key process parameters with regard to the formation of magnesium oxychloride fibers we can also demonstrate the great potential of $\mathrm{MgO}$ nanocubes as a model system for the elucidation of growth of anisotropic nanostructures ${ }^{29}$ the associated heterogeneous chemistry and, ultimately, the evolution of resulting microstructures. An important aspect relates to the localization of these transformation processes, i.e. the base of $\mathrm{Mg}_{3}(\mathrm{OH})_{5} \mathrm{Cl} \cdot 4 \mathrm{H}_{2} \mathrm{O}$ fiber growth. Spatially confined reaction media ${ }^{30}$ both in the $\mu \mathrm{m}$ range (agglomerates of functionalized $\mathrm{MgO}$ nanocubes) as well as in the range of few nanometers (thin liquid water films for ion transport and reaction) were shown to determine the transformation and microstructure evolution. The here presented mechanistic insights are important for the chemical development of new nanostructured phases. ${ }^{31,32}$ It can be applied to a variety of chemical approaches that involve metastable and surface functionalized nanomaterials and superficial water which originates from humid air and acts as a reactant and reaction medium for materials' transformation.

\section{Acknowledgements}

We are grateful for substantial support from the German Research Foundation (DFG-DI 1613/2-1). O. D. and A. S. acknowledge the support of the German Research Foundation (DFG), which, within the framework of its "Excellence Initiative", supports the cluster of Excellence "Engineering of Advanced Materials" at the University of Erlangen-Nürnberg.

\section{Notes and references}

1 T. Berger and O. Diwald, Defects in Metal Oxide Nanoparticle Powders, in Defects at Oxide Surfaces, ed. J. Jupille and G. Thornton, Springer International Publishing, 2015, p. 273.

2 I. A. Mudunkotuwa and V. H. Grassian, J. Environ. Monit., 2011, 13(5), 1135.

3 Y. Wang, J. He, C. Liu, W. H. Chong and H. Chen, Angew. Chem., Int. Ed., 2015, 54(7), 2022.

4 Nanoparticles from the Gasphase: Formation, Structure, Properties, ed. A. Lorke, M. Winterer, R. Schmechel and C. Schulz, Springer Berlin Heidelberg, Berlin, Heidelberg, 2012.

5 J. T. Newberg, T. M. McIntire and J. C. Hemminger, J. Phys. Chem. A, 2010, 114(35), 9480.

6 G. Rubasinghege and V. H. Grassian, Chem. Commun., 2013, 49(30), 3071.

7 S. Garde and M. L. Schlossman, MRS Bull., 2014, 39(12), 1051.

8 W. Stumm, L. Sigg and B. Sulzberger, Chemistry of the solidwater interface: Processes at the mineral-water and particlewater interface in natural systems, Wiley, New York, 1992.
9 M. Henderson, Surf. Sci. Rep., 2002, 46(1-8), 1.

10 P. Jungwirth, B. J. Finlayson-Pitts and D. J. Tobias, Chem. Rev., 2006, 106(4), 1137.

11 P. Fenter and S. S. Lee, MRS Bull., 2014, 39(12), 1056.

12 T. P. Vinod, N. Froumin, D. Mogiliansky, L. Zeiri, V. Ezersky and R. Jelinek, ChemPhysChem, 2014, 15(14), 3026.

13 H. Zhang, Y. Li, P. Liu, Y. Li, D. Yang, H. Yang and H. Zhao, Chem.-Eur. J., 2012, 18(17), 5165.

14 A. Gheisi, A. Sternig, M. Rangus, G. Redhammer, M. Hartmann and O. Diwald, Cryst. Growth Des., 2014, 14, 4236.

15 A. Sternig, S. Klacar, J. Bernardi, M. Stöger-Pollach, H. Grönbeck and O. Diwald, J. Phys. Chem. C, 2011, 115(32), 15853.

16 A. Sternig, D. Koller, N. Siedl, O. Diwald and K. McKenna, J. Phys. Chem. C, 2012, 116(18), 10103.

17 O. Diwald, M. Sterrer and E. Knözinger, Phys. Chem. Chem. Phys., 2002, 4(12), 2811.

18 M. Müller, S. Stankic, O. Diwald, E. Knözinger, P. V. Sushko, P. E. Trevisanutto and A. L. Shluger, J. Am. Chem. Soc., 2007, 129(41), 12491.

19 S. O. Baumann, J. Schneider, A. Sternig, D. Thomele, S. Stankic, T. Berger, H. Grönbeck and O. Diwald, Langmuir, 2015, 31(9), 2770.

20 S. Stankic, M. Müller, O. Diwald, M. Sterrer, E. Knözinger and J. Bernardi, Angew. Chem., Int. Ed., 2005, 44(31), 4917.

21 C. Weidenthaler, Nanoscale, 2011, 3(3), 792.

22 A. Weibel, R. Bouchet, F. Boulc' and P. Knauth, Chem. Mater., 2005, $17(9), 2378$.

23 Different to $\mathrm{HCl}, \mathrm{SiCl}_{4}$ can be applied in water free form via the gasphase and - important for the highly sensitive surface reaction reported here - exhibits a lower reactivity than $\mathrm{TiCl}_{4}$.

24 We did not perform a systematic study on the time dependence of this transformation process, which is beyond the scope of this study. However, from XRD measurements we learned that the transformation from $\mathrm{MgO}$ periclase either into Brucite or into oxychloride fibers has occurred within the first 3 days. After this time some $\mathrm{MgO}$ derived remnants that have escaped XRD detection were identified by TEM. For this reason a total exposure time of 14 days was selected to guarantee the quantitative transformation of all $\mathrm{MgO}$ based nanostructures involved as judged from TEM analysis.

25 K. Sugimoto, R. E. Dinnebier and T. Schlecht, Acta Crystallogr., Sect. B: Struct. Sci., 2007, 63(6), 805.

26 T. Runčevski, R. E. Dinnebier and D. Freyer, Z. Anorg. Allg. Chem., 2014, 640(1), 100.

27 T. Runčevski, C. Wu, H. Yu, B. Yang, R. E. Dinnebier and H. Jennings, J. Am. Ceram. Soc., 2013, 96(11), 3609.

28 K. P. McKenna, D. Koller, A. Sternig, N. Siedl, N. Govind, P. V. Sushko and O. Diwald, ACS Nano, 2011, 5(4), 3003.

29 S. O. Baumann, C. Liu, M. J. Elser, A. Sternig, N. Siedl, T. Berger and O. Diwald, Chem.-Eur. J., 2013, 19, 10235.

30 M. Fröba and A. Reller, Prog. Solid State Chem., 1999, 27(1), 1. 31 S. Mann, Nat. Mater., 2009, 8(10), 781.

32 F. C. Meldrum and H. Cölfen, Nanoscale, 2010, 2(11), 2326. 